Brown-HET-1579

\title{
Correlation Functions in Non-Relativistic Holography
}

\author{
Anastasia Volovich ${ }^{1}$ and Congkao $\mathrm{Wen}^{1}$ \\ ${ }^{1}$ Brown University, Providence, Rhode Island 02912, USA
}

\begin{abstract}
Recently constructed gravity solutions with Schrödinger symmetry provide a new example of AdS/CFT-type dualities for the type of non-relativistic field theories relevant to certain cold atom systems. In this paper we use the gravity side to calculate $n$-point correlation functions of scalar fields by reducing the computation to that in ordinary AdS space via a particular Fourier transform. We evaluate the relevant integrals for 3- and 4-point functions and show that the results are consistent with the requirements of Schrödinger invariance, the implications of which we also work out for general $n$-point functions.
\end{abstract}




\section{INTRODUCTION}

Gauge/string duality is a central theme of modern research in string theory [1, 2, 3]. As a strong/weak coupling duality it provides us with a wealth of data useful for studying interesting field theories at strong coupling where other methods fail. Its applications currently range from nuclear physics to plasma physics to tabletop condensed matter systems [4].

Until recently the study of gauge/gravity duality has been limited to relativistic field theories. However many non-relativistic field theories clearly play important roles in various physical systems. An example of a system with non-relativistic conformal symmetry is a system of fermions at unitarity which can be realized experimentally in certain cold atom systems [5]. This theory is invariant under a non-relativistic conformal group called the Schrödinger group, and it is natural to wonder if there exists a gravity dual which would allow us to use the AdS/CFT correspondence to learn more about these systems.

Recently a new exciting example of such duality has emerged. The simplest geometries with Schrödinger group isometry have been constructed in [6, 7], leading to what is sometimes referred to as the AdS/cold atom correspondence or non-relativistic AdS/CFT. Excitingly, these geometries have been embedded in string theory in [8, 9, 10]. These papers have opened up a new avenue for studying non-relativistic conformal field theories (NRCFTs) at strong coupling. See [11] for recent studies of various aspects of non-relativistic $\mathrm{AdS} / \mathrm{CFT}$.

The holographic dictionary has been developed in [6, 7] and the two-point function of a scalar field was recovered from a gravity calculation in [6, 7]. Unlike more familiar applications of AdS/CFT where the boundary is one dimension less than the bulk, here the boundary theory has two fewer dimensions than the bulk [6, 7].

In this paper we consider higher point correlation functions in the gravity theory. We employ the trick introduced in [12] where the free massive Schrödinger equation was reduced to a non-massive Klein-Gordon equation via Fourier transform with respect to the mass. Applying the same trick to the solution of the scalar wave equation in the gravity background for non-relativistic field theory, we reduce the wave equation to that in ordinary AdS space. The computation of correlation functions in the bulk then reduces to performing a particular Fourier transform of AdS correlators. We explicitly evaluate the relevant integrals for three- and four-point functions and show that the results are indeed consistent 
with the requirements of Schrödinger invariance, the implications of which we work out for general $n$-point functions. The same integral for three-point function appeared in [12], where these nonrelativistic three-point functions where identified with response functions in Martin-Siggia-Rose theory, but the integral has not been explicitly evaluated there.

The paper is organized as follows. In section 2 we briefly review the necessary results from non-relativistic conformal field theories and discuss the constraints that Schrödinger symmetry places on $n$-point functions of primary operators. In section 3 we compute the bulk-to-bulk and bulk-to-boundary propagators for a scalar field. In section 4 we compute three- and four-point functions on the gravity side and compare them with the corresponding correlators in NRCFT. In appendix we explicitly evaluate the gravity bulk integrals which appear.

Note Added. While this paper was in preparation the paper [13] appeared which also considers three-point function in non-relativistic AdS/CFT.

\section{CORRELATION FUNCTIONS WITH SCHRÖDINGER SYMMETRY}

In this section we first review, following [14], a few of the most essential features of the Schrödinger symmetry. We then address the question of what can be said about the structure of general $n$-point functions just based on requiring invariance under this symmetry group, generalizing some of the discussion of [15, 16].

The generators of the Schrödinger group are the number operator (or the 'mass' operator) $M$, the dilatation operator $D$ which generates scale transformations

$$
D:(\vec{x}, t) \rightarrow\left(\lambda \vec{x}, \lambda^{2} t\right)
$$

the momentum and energy operators $P_{i}$ and $H$ which generate space and time translations

$$
P_{i}: \vec{x} \rightarrow \vec{x}+\vec{a}, \quad H: T \rightarrow t+a,
$$

the angular momentum operators $M_{i j}$ which generate spatial rotations

$$
M_{i j}: \vec{x} \rightarrow R \vec{x}
$$

the generators $K_{i}$ of Galilean boosts

$$
K_{i}: \vec{x} \rightarrow \vec{x}-\vec{v} t
$$


and finally the generator $C$ of special conformal transformations

$$
C:(\vec{x}, t) \rightarrow\left(\frac{\vec{x}}{1+a t}, \frac{t}{1+a t}\right),
$$

which can alternatively be expressed as $C=T_{1} H T_{1}$ in terms of

$$
T_{1}:(\vec{x}, t) \rightarrow\left(\frac{\vec{x}}{t}, \frac{1}{t}\right)
$$

For an operator $\mathcal{O}$ of definite conformal dimension $\Delta_{\mathcal{O}}$ we have [14]

$$
\begin{aligned}
{\left[D,\left[K_{i}, \mathcal{O}\right]\right] } & =i\left(\Delta_{\mathcal{O}}-1\right)\left[K_{i}, \mathcal{O}\right] \\
{[D,[C, \mathcal{O}]] } & =i\left(\Delta_{\mathcal{O}}-2\right)[C, \mathcal{O}]
\end{aligned}
$$

Since $K_{i}$ and $C$ evidently lower the dimension of any operator we can define a primary operator to be one which satisfies the conditions

$$
\left[K_{i}, \mathcal{O}\right]=0, \quad[C, \mathcal{O}]=0
$$

Starting with a primary operator one can build up a tower of descendants by repeated commutation with $H$ and/or $P_{i}$, which always raises the dimension.

Let us now consider what can be said in general about the structure of an $n$-point correlation function

$$
A_{n}(1, \ldots, n) \equiv\left\langle\mathcal{O}_{1}\left(\vec{x}_{1}, t_{1}\right) \cdots \mathcal{O}_{n}\left(\vec{x}_{n}, t_{n}\right)\right\rangle
$$

of primary operators. First we can use translation invariance to reduce the $n$ coordinates $\left(\vec{x}_{i}, t_{i}\right)$ to $(n-1)$ independent coordinates $\left(\vec{x}_{i j}, t_{i j}\right)$, where $t_{i j}=t_{i}-t_{j}$ and $\vec{x}_{i j}=\left(\vec{x}_{i}-\vec{x}_{j}\right)$. Since the $(n-1)$ time variables are automatically invariant under the $K_{i}$ we can build conformally invariant variables from them just as in more familiar relativistic CFTs. Specifically, scale invariance implies that only ratios such as $t_{i j} / t_{k l}$ may appear, while invariance under special conformal invariance allows only the familiar cross-ratios of the form $t_{i j} t_{k l} / t_{i k} t_{j l}$, of which $n(n-3) / 2$ are independent [16].

Now consider the $(n-1)$ vectors $\vec{x}_{i j}$, from which we can build a total of $n(n-1) / 2$ independent scalars $\vec{x}_{i j} \cdot \vec{x}_{k l}$. Then we use $(n-1)$ Galilean boosts similar to the procedure in [16] to reduce this number to $(n-1)(n-2) / 2$. Actually the resulting independent conformally invariant variables can be parametrized as

$$
v_{i j}=\frac{\left(\vec{x}_{i n} t_{j n}-\vec{x}_{j n} t_{i n}\right)^{2}}{2 t_{i j} t_{i n} t_{j n}}=\frac{1}{2}\left(\frac{x_{j n}^{2}}{t_{j n}}-\frac{x_{i n}^{2}}{t_{i n}}+\frac{x_{i j}^{2}}{t_{i j}}\right), \quad i<j<n,
$$


and this is the form in which they will appear naturally from the AdS calculations in the next section. One can easily check the $v_{i j}$ are indeed invariant under all of the Schrödinger group generators.

So in general an $n$-point function will always be allowed to have arbitrary functional dependence on a total of $n^{2}-3 n+1$ Shrödinger-invariant variables. The functional dependence on the remaining, non-conformally invariant variables, can be determined by solving the analogue of the conformal Ward identities, which are differential equations expressing the constraints of the symmetry on correlation functions.

For example it is well-known [15] that the 2-point function is completely fixed up to an overall constant to the form

$$
A_{2}(1,2)=c \delta_{\Delta_{1}, \Delta_{2}} t_{12}^{-\Delta_{1}} e^{\frac{i M}{2} \frac{x_{12}^{2}}{t_{12}}}
$$

The non-relatvistic 3-point function has been shown [15] to be determined as

$$
A_{3}(1,2,3)=\prod_{i<j} t_{i j}^{\Delta / 2-\left(\Delta_{i}+\Delta_{j}\right)} e^{i\left(\frac{M_{1}}{2} \frac{x_{13}^{2}}{t_{13}}+\frac{M_{2}}{2} \frac{x_{23}^{2}}{t_{23}}\right)} F\left(v_{12}\right)
$$

where $F$ is an arbitrary function. Analagously, we find that the general form of the 4-point function is

$$
A_{4}(1,2,3,4)=\prod_{i<j} t_{i j}^{\Delta / 6-\left(\Delta_{i}+\Delta_{j}\right) / 2} e^{i\left(\frac{M_{1}}{2} \frac{x_{14}^{2}}{t_{14}}+\frac{M_{2}}{2} \frac{x_{24}^{2}}{t_{24}}+\frac{M_{3}}{2} \frac{x_{34}^{2}}{t_{34}}\right)} F\left(\frac{t_{12} t_{34}}{t_{14} t_{23}}, \frac{t_{12} t_{34}}{t_{13} t_{24}}, v_{12}, v_{13}, v_{23}\right)
$$

where $\Delta=\sum_{i} \Delta_{i}$. It is a simple exercise to check that this satisfies the relevant conformal Ward identities.

\section{NON-RELATIVISTIC ADS/CFT}

The metric with Schrödinger group isometry constructed in [6, 7] is

$$
d s^{2}=L^{2}\left(-\frac{d t^{2}}{r^{4}}+\frac{2 d \xi d t+d \vec{x}^{2}}{r^{2}}+\frac{d r^{2}}{r^{2}}\right)
$$

where $\vec{x}=x_{i}$ and $i=1,2 \ldots d$. Let us consider a massive scalar field in this background. The wave equation is

$$
\left(\nabla^{2}-m_{0}^{2}\right) \phi=\left(r^{d+3} \partial_{r}\left(\frac{1}{r^{d+1}} \partial_{r}\right)+r^{2}\left(2 \partial_{\xi} \partial_{t}+r^{-2} \partial_{\xi}^{2}+\partial_{i}^{2}\right)-m_{0}^{2}\right) \phi\left(r, \xi, x_{i}, t\right)=0 .
$$

Because $\xi$ is a compact direction we can use 


$$
\phi\left(r, \xi, x_{i}, t\right)=e^{i M \xi} \phi_{M}\left(r, x_{i}, t\right)
$$

to rewrite the equation (3.2) on the Fourier modes as

$$
\left(r^{d+3} \partial_{r}\left(\frac{1}{r^{d+1}}\right)+r^{2}\left(2 i M \partial_{t}+\partial_{i}^{2}\right)-m^{2}\right) \phi_{M}\left(r, x_{i}, t\right)=0,
$$

where $m^{2}=M^{2}+m_{0}^{2}$. In order to make the equation more symmetric we can introduce a new coordinate $\eta$ by

$$
\phi_{M}\left(r, x_{i}, t\right)=\int d \eta e^{-i M \eta} \psi\left(r, \eta, x_{i}, t\right) .
$$

This is the same trick that was introduced in [12], where the free massive Schrödinger equation was reduced to a non-massive Klein-Gordon equation via Fourier transform with respect to the mass.

If $\psi\left(r, \eta= \pm \infty, x_{i}, t\right) \rightarrow 0$ we can integrate by parts so that (3.4) becomes effectively

$$
\left(r^{d+3} \partial_{r}\left(\frac{1}{r^{d+1}} \partial_{r}\right)+r^{2}\left(2 \partial_{\eta} \partial_{t}+\partial_{i}^{2}\right)-m^{2}\right) \psi\left(r, \eta, x_{i}, t\right)=0,
$$

and we can further simplify the equation by introducing the $\chi$ coordinates according to

$$
\begin{aligned}
t & =\sqrt{\frac{1}{2}}\left(\chi_{0}-i \chi_{n+1}\right), \\
\eta & =\sqrt{\frac{1}{2}}\left(\chi_{0}+i \chi_{n+1}\right), \\
x_{i} & =\chi_{i} .
\end{aligned}
$$

Then the wave equation (3.1) becomes identical to that of a scalar field in a Euclidean AdS background

$$
\left(r^{d+3} \partial_{r}\left(\frac{1}{r^{d+1}} \partial_{r}\right)+r^{2}\left(\partial_{\chi_{i}}^{2}+\partial_{\chi_{0}}^{2}+\partial_{\chi_{n+1}}^{2}\right)-m^{2}\right) \psi\left(r, \chi_{i}\right)=0 .
$$

We will use exactly same strategy in the next section to compute the bulk-to-boundary and bulk-to-bulk propagators in the background (3.1).

\section{A. The bulk-to-boundary propagator and two-point function}

The boundary of the background (3.1) is at $r=0$ and the generator associated with translations along the compact $\xi$ direction idenfied as the mass operator $M=i \partial_{\xi}$, So a $(d+3)$-dimensional bulk theory is dual to a $(d+1)$-dimension boundary theory [6, 7$]$. 
Near the boundary, solutions of the scalar wave equation behave like

$$
\phi \rightarrow r^{(d+2)-\Delta} \phi_{0}
$$

where

$$
\Delta=1+\frac{d}{2}+\sqrt{\left(1+\frac{d}{2}\right)^{2}+m_{0}^{2}+M^{2}}
$$

is related to the scaling dimension of the source $\mathcal{O}$ in the boundary theory. We will use the usual AdS/CFT recipe [2, 3] to calculate correlation functions of $\mathcal{O}$.

In order to compute the two-point function of the boundary operators we have to calculate the on-shell action of a massive scalar field for a solution of the classical equation of motion

$$
\nabla^{2} \phi_{M}=m_{0}^{2} \phi_{M}
$$

subject to the boundary condition

$$
\lim _{r \rightarrow 0} \phi_{M}(r, t, \vec{x})=r^{(d+2)-\Delta} \phi_{0}(t, \vec{x})
$$

where $\Delta$ is given in (3.10). We used the compactness of $\xi(3.3)$ in order not to write the explicit $\xi$ dependence focusing on a Fourier mode $\phi_{M}$.

The relevant solution of $(3.11)$ is given by

$$
\phi_{M}(r, \vec{x}, t)=\int d \vec{x}_{1} d t_{1} K\left(r, \vec{x}, t ; \vec{x}_{1}, t_{1}\right) \phi_{0}\left(\vec{x}_{1}, t_{1}\right)
$$

where $K\left(r, \vec{x}, t ; \vec{x}_{1}, t_{1}\right)$ is the bulk-to-boundary propagator for metric (3.1)

$$
K\left(r, \vec{x}, t ; \vec{x}_{1}, t_{1}\right)=\frac{i\left(\frac{M}{2}\right)^{\Delta-1} e^{-i \pi \Delta / 2}}{\pi^{\frac{d}{2}} \Gamma\left(\Delta-\left(\frac{d}{2}+1\right)\right)} \theta\left(t-t_{1}\right)\left(\frac{r}{t-t_{1}}\right)^{\Delta} e^{\frac{i}{2} M(1+i \epsilon) \frac{r^{2}+\left(x-x_{1}\right)^{2}}{\left(t-t_{1}\right)}}
$$

where $\epsilon$ is the regulator. As expected this heat kernel is the solution of (3.11) which in the limit $r \rightarrow 0$ behaves as a delta function

$$
r^{\Delta-(d+2)} K\left(r, \vec{x}, t ; \vec{x}_{1}, t_{1}\right) \rightarrow \delta^{d}\left(x-x_{1}\right) \delta\left(t-t_{1}\right) .
$$

Let us now show how the bulk-to-boundary propagator (3.14) may be derived by using a trick similar to the one described in the previous section. Using (3.3) and (3.5), we get the following representation for the bulk-to-boundary propagator,

$$
K\left(r, \vec{x}, t ; \vec{x}_{1}, t_{1}\right)=\int d \eta e^{-i M \eta} K\left(r, \eta, \vec{x}, t ; \vec{x}_{1}, t_{1}\right)
$$


where $K\left(r, \eta, \vec{x}, t ; \vec{x}_{1}, t_{1}\right)$ is the bulk-to-boundary propagator in Euclidean AdS space (3.8) [3, 18, 19]

$$
K\left(r, \eta, \vec{x}, t ; \vec{x}_{1}, t_{1}\right)=c_{\Delta}\left(\frac{r}{r^{2}+2\left(t-t_{1}\right) \eta+\left(x-x_{1}\right)^{2}}\right)^{\Delta},
$$

with $\Delta$ given by $(3.10)$ and $c_{\Delta}=\frac{i \Gamma(\Delta)}{\pi^{1+\frac{d}{2}} \Gamma\left(\Delta-\left(\frac{d}{2}+1\right)\right)}$.

The integral (3.16) is not well-defined as it stands, so we introduce the usual regulator $\epsilon$ by $t \rightarrow t(1-i \epsilon)$ which is equivalent to taking $t \rightarrow-i t$ as was done in [12]. This leads to integral representation of $K\left(r, \vec{x}, t ; \vec{x}_{1}, t_{1}\right)$

$$
c_{\Delta} \int_{\mathbf{R}} d \eta e^{-i M \eta}\left(\frac{r}{r^{2}+2\left(t-t_{1}\right)(1-i \epsilon) \eta+\left(x-x_{1}\right)^{2}}\right)^{\Delta} .
$$

It will become useful for computing higher point correlation functions in the next section. The result (3.14) follows after performing the $\eta$ integral here.

Returning now to the solution (3.13), we find that when evaluating the action of this solution only a boundary term contributes

$$
S[\phi]=\lim _{r \rightarrow 0} \int d^{d} \vec{x} d t r^{-1-d} \phi \partial_{r} \phi,
$$

and in this manner we find

$$
S[\phi]=a_{\Delta} \int d^{d} \overrightarrow{x_{1}} d t_{1} d^{d} \overrightarrow{x_{2}} d t_{2} \phi_{0}\left(\overrightarrow{x_{1}}, t_{1}\right) \theta\left(t_{1}-t_{2}\right)\left(\frac{1}{t_{1}-t_{2}}\right)^{\Delta} e^{i \frac{M}{2} \frac{\left(x_{1}-x_{2}\right)^{2}}{\left(t_{1}-t_{2}\right)}} \phi_{0}\left(\overrightarrow{x_{2}}, t_{2}\right),
$$

where $^{1}$

$$
a_{\Delta}=\frac{i \Delta\left(\frac{M}{2}\right)^{\Delta-1} e^{-i \pi \Delta / 2}}{\pi^{\frac{d}{2}} \Gamma\left(\Delta-\left(\frac{d}{2}+1\right)\right)} .
$$

Thus the boundary action correctly reproduces the two-point function in non-relativistic CFT (2.11)

$$
A_{2}(1,2)=\frac{i \Delta\left(\frac{M}{2}\right)^{\Delta-1} e^{-i \pi \Delta / 2}}{\pi^{\frac{d}{2}} \Gamma\left(\Delta-\left(\frac{d}{2}+1\right)\right)} \theta\left(t_{1}-t_{2}\right)\left(\frac{1}{t_{1}-t_{2}}\right)^{\Delta} e^{i \frac{M}{2} \frac{\left(x_{1}-x_{2}\right)^{2}}{\left(t_{1}-t_{2}\right)}} .
$$

This was also found in [7].

\footnotetext{
${ }^{1}$ The careful treatment of the $r \rightarrow 0$ limit amounts to multiplying this result by an additional factor of $\frac{2 \Delta-(d+2)}{\Delta}$ [18].
} 


\section{B. The bulk-to-bulk propagator}

The bulk-to-bulk propagator $G_{M}\left(r, \vec{x}, t ; r_{1}, \vec{x}_{1}, t_{1}\right)$ for a scalar field in background (3.1) satisfies

$$
\begin{aligned}
\left(r^{d+3} \partial_{r}\left(\frac{1}{r^{d+1}} \partial_{r}\right)+r^{2}\left(2 i M \partial_{t}+\partial_{i}^{2}\right)-\left(m_{0}^{2}+M^{2}\right)\right) G_{M}\left(r, \vec{x}, t ; r_{1}, \vec{x}_{1}, t_{1}\right) & \\
& =r^{d+3} \delta\left(r-r_{1}\right) \delta^{d}\left(\vec{x}-\vec{x}_{1}\right) \delta\left(t-t_{1}\right) .
\end{aligned}
$$

Here we again used the compactness of $\xi$ and wrote an equation for the corresponding Fourier mode, in terms of which the full bulk-to-bulk propagator is

$$
G\left(r, \xi, \vec{x}, t ; r_{1}, \xi_{1}, \vec{x}_{1}, t_{1}\right)=e^{i M\left(\xi-\xi_{1}\right)} G_{M}\left(r, \vec{x}, t ; r_{1}, \vec{x}_{1}, t_{1}\right) .
$$

If we introduce the coordinate $\eta$ by $^{2}$

$$
\delta\left(M-M_{1}\right) G_{M}\left(r, \vec{x}, t ; r_{1}, \vec{x}_{1}, t_{1}\right)=\int d \eta d \eta_{1} e^{-i M \eta+i M_{1} \eta_{1}} G_{M}\left(r, \eta, \vec{x}, t ; r_{1}, \eta_{1}, \vec{x}_{1}, t_{1}\right),
$$

we then find that $G_{M}\left(r, \eta, \vec{x}, t ; r_{1}, \eta_{1}, \vec{x}_{1}, t_{1}\right)$ satisfies

$$
\begin{aligned}
\left(r^{d+3} \partial_{r}\left(\frac{1}{r^{d+1}} \partial_{r}\right)+r^{2}\left(2 \partial_{\eta} \partial_{t}+\partial_{i}^{2}\right)-\right. & \left.m^{2}\right) G_{M}\left(r, \eta, \vec{x}, t ; r_{1}, \eta_{1}, \vec{x}_{1}, t_{1}\right) \\
& =r^{d+3} \delta\left(\eta-\eta_{1}\right) \delta\left(r-r_{1}\right) \delta^{d}\left(\vec{x}-\vec{x}_{1}\right) \delta\left(t-t_{1}\right) .
\end{aligned}
$$

This in turn is nothing other than the equation for bulk-to-bulk propagator in $A d S$, whose solution is [17, 19]

$$
G_{M}\left(r, \eta, \vec{x}, t ; r_{1}, \eta_{1}, \overrightarrow{x_{1}}, t_{1}\right)=\tilde{C}_{\Delta}(2 z)^{\Delta}{ }_{2} F_{1}\left(\frac{\Delta}{2}, \frac{\Delta}{2}+\frac{1}{2} ; \Delta-\frac{d}{2}, z^{2}\right),
$$

where

$$
\begin{aligned}
\tilde{C}_{\Delta} & =\frac{i \Gamma(\Delta) \Gamma\left(\Delta-\frac{d}{2}-\frac{3}{2}\right)}{(4 \pi)^{(d+3) / 2} \Gamma(2 \Delta-d-1)}, \\
z & =\frac{2 r r_{1}}{r^{2}+r_{1}^{2}+\left(\vec{x}-\overrightarrow{x_{1}}\right)^{2}+2\left(\eta-\eta_{1}\right)\left(t-t_{1}\right)}
\end{aligned}
$$

\footnotetext{
${ }^{2}$ It might appear that we have introduced one more unwanted parameter $M_{1}$, but because $G\left(r, \eta, \vec{x}, t ; r_{1}, \eta_{1}, \vec{x}_{1}, t_{1}\right)$ is invariant under translations in $\eta$, the integral will force $M=M_{1}$. That is why there is a $\delta\left(M-M_{1}\right)$ on the left hand side.
} 
By changing variables we find that bulk-to-bulk propagator may be expressed as

$$
\begin{aligned}
& G_{M}\left(r, \vec{x}, t ; r_{1}, \vec{x}_{1}, t_{1}\right)= \\
& \tilde{C}_{\Delta} e^{\frac{i}{2} M(1+i \epsilon) \frac{y^{2}}{t-t_{1}}}\left(\frac{2 r r_{1}}{t-t_{1}}\right)^{\Delta} \int_{\mathcal{C}} d u e^{-i M u} u^{-\Delta_{2}} F_{1}\left(\frac{\Delta}{2}, \frac{\Delta}{2}+\frac{1}{2} ; \Delta-\frac{d}{2} ;\left(\frac{r r_{1}}{\left(t-t_{1}\right) u}\right)^{2}\right)
\end{aligned}
$$

where $y^{2}=r^{2}+r_{1}^{2}+\left(\vec{x}-\vec{x}_{1}\right)^{2}$ and $\mathcal{C}$ is $\mathbf{R}+\frac{y^{2}}{2\left(t-t_{1}\right)} i \epsilon$.

Using

$$
{ }_{2} F_{1}(a, b ; c ; z)=\sum_{n=0}^{\infty} \frac{(a)_{n}(b)_{n}}{(c)_{n} n !} z^{n}
$$

we have

$$
\begin{aligned}
& \int_{\mathcal{C}} d u e^{-i M u} u^{-\Delta}{ }_{2} F_{1}\left(a, b ; c ;(k / u)^{2}\right)= \\
& =\sum_{n=0}^{\infty} \frac{(a)_{n}(b)_{n}}{(c)_{n} n !}\left(k^{2}\right)^{n} \int_{\mathcal{C}} d u e^{-i M u} u^{-(\Delta+2 n)}= \\
& =2 \pi M^{\Delta-1} e^{-i \pi \Delta / 2} \sum_{n=0}^{\infty} \frac{(a)_{n}(b)_{n}}{(c)_{n} n !} \frac{(-i M k)^{2 n}}{\Gamma(\Delta+2 n)}= \\
& =\frac{2 \pi M^{\Delta-1} e^{-i \pi \Delta / 2}}{\Gamma(\Delta)}{ }_{2} F_{3}\left(a, b ; c, \frac{1}{2}+\frac{\Delta}{2}, \frac{\Delta}{2} ;-\frac{M^{2} k^{2}}{4}\right) .
\end{aligned}
$$

Putting everything together we find that the bulk-to-bulk propagator is given by

$$
G_{M}\left(r, \vec{x}, t ; r_{1}, \vec{x}_{1}, t_{1}\right)=K_{\Delta} \theta\left(t-t_{1}\right) e^{\frac{i M(1+i \epsilon)}{2} \frac{y^{2}}{t-t_{1}}}\left(\frac{r r_{1}}{t-t_{1}}\right)^{\frac{d}{2}+1} J_{\Delta-\frac{d}{2}-1}\left(\frac{M r r_{1}}{t-t_{1}}\right)
$$

where $J$ is the Bessel function and the constant is

$$
K_{\Delta}=\frac{\pi \tilde{C}_{\Delta} M^{d / 2} 2^{2 \Delta-d / 2} e^{-i \pi \Delta / 2} \Gamma(\Delta-d / 2)}{\Gamma(\Delta)}
$$

\section{HIGHER-POINT CORRELATION FUNCTIONS}

\section{A. Three-point functions}

Now let us calculate boundary three-point functions. We consider bulk interaction ver-

tices of the form $L_{1}=\phi^{3}$ and $L_{2}=\phi \partial_{\mu} \phi \partial^{\mu} \phi$. The corresponding three-point functions are respectively

$$
\begin{aligned}
& A_{3}(1,2,3)=\int \frac{d r d^{d} x d t d \xi}{r^{d+3}} K_{1}^{*}\left(r, \xi, \vec{x}, t ; \vec{x}_{1}, t_{1}\right) K_{2}^{*}\left(r, \xi, \vec{x}, t ; \vec{x}_{2}, t_{2}\right) K_{3}\left(r, \xi, \vec{x}, t ; \vec{x}_{3}, t_{3}\right), \\
& A_{3}^{\prime}(1,2,3)=\int \partial_{\mu} K_{1}^{*} \partial^{\mu} K_{2}^{*} K_{3} .
\end{aligned}
$$


Using the representation (3.16) of the bulk-to-boundary propagator, changing variables and using number conservation (which comes from the $\xi$ integral) we find

$$
\begin{gathered}
A_{1}(1,2,3)=c_{1} \int d \alpha^{\prime} d \beta^{\prime} e^{-i M_{1} \alpha^{\prime}} e^{-i M_{2} \beta^{\prime}} \int \frac{d r d^{d} x d t d \gamma}{r^{d+3}}\left(\frac{r}{r^{2}+2\left(t-t_{1}\right)\left(\gamma-\alpha^{\prime}\right)+\left(x-x_{1}\right)^{2}}\right)^{\Delta_{1}} \\
\left.\times\left(\frac{r}{r^{2}+2\left(t-t_{2}\right)\left(\gamma-\beta^{\prime}\right)+\left(x-x_{2}\right)^{2}}\right)^{\Delta_{2}}\left(\frac{r}{r^{2}+2\left(t-t_{3}\right) \gamma+\left(x-x_{3}\right)^{2}}\right)^{\Delta_{3}}, \quad, 4.3\right)
\end{gathered}
$$

where $c_{1}=\bar{c}_{\Delta_{1}} \bar{c}_{\Delta_{2}} c_{\Delta_{3}}$.

Using the known relativistic AdS/CFT [17] result and introducing the regulator, we can perform the integral to arrive at

$$
A_{1}(1,2,3)=a_{1}\left(t_{12}\right)^{-\Delta_{12,3} / 2}\left(t_{23}\right)^{-\Delta_{23,1} / 2}\left(t_{13}\right)^{-\Delta_{13,2} / 2} e^{i\left(\frac{M_{1}}{2} \frac{x_{13}^{2}}{t_{13}}+\frac{M_{2}}{2} \frac{x_{23}^{2}}{t_{23}}\right)} I
$$

where

$$
a_{1}=-\frac{\Gamma\left[\frac{1}{2}(\Delta-(d+2))\right] M_{1}^{\Delta_{1}-1} M_{2}^{\frac{\Delta_{23,1}}{2}-1} e^{-i \pi \Delta / 4}}{2^{\Delta / 2-1} \pi^{d} \Gamma\left[\Delta_{1}-\frac{d}{2}-1\right] \Gamma\left[\Delta_{2}-\frac{d}{2}-1\right] \Gamma\left[\Delta_{3}-\frac{d}{2}-1\right]}
$$

and

$$
\begin{aligned}
I & =\frac{1}{C} \int_{\mathcal{C}_{1}} d u \int_{\mathcal{C}_{2}} d w e^{-i M_{1} u} e^{-i M_{2} w} \frac{1}{\left(u-w+v_{12}(1+i \epsilon)\right)^{\Delta_{12,3} / 2}} \frac{1}{w^{\Delta_{23,1} / 2} u^{\Delta_{13,2} / 2}} \\
& =\theta\left(t_{23}\right) \theta\left(t_{13}\right) B\left(\frac{\Delta_{12,3}}{2}, \frac{\Delta_{13,2}}{2}\right) \Phi_{1}\left(\frac{\Delta_{12,3}}{2}, \frac{\Delta_{23,1}}{2}-1, \Delta_{1},-\frac{M_{1}}{M_{2}}, i M_{1} v_{12}\right)
\end{aligned}
$$

where the contour $\mathcal{C}_{j}$ is $\left(\mathbf{R}+i \frac{\vec{x}_{j 3}^{2}}{2 t_{j 3}} \epsilon\right)$ and

$$
\begin{aligned}
v_{12} & =\frac{1}{2}\left(\frac{x_{12}^{2}}{t_{12}}+\frac{x_{23}^{2}}{t_{23}}-\frac{x_{13}^{2}}{t_{13}}\right), \\
\Delta & =\sum_{i} \Delta_{i}, \quad \Delta_{i j, k}=\Delta_{i}+\Delta_{j}-\Delta_{k} \\
C & =\frac{4 \pi^{2} M_{1}^{\Delta_{1}-1} M_{2}^{\frac{\Delta_{23,1}}{2}-1} e^{-\frac{i \pi}{4} \Delta}}{\Gamma\left[\frac{\Delta_{12,3}}{2}\right] \Gamma\left[\frac{\Delta_{23,1}}{2}\right] \Gamma\left[\frac{\Delta_{13,2}}{2}\right]} .
\end{aligned}
$$

The beta function $B(\nu, \lambda)$ and the confluent hypergeometric $\Phi_{1}(\alpha, \beta, \gamma, x, y)$ are defined in (A9) and (A10) respectively.

Similarly, for the second type of interaction we find

$$
A_{2}(1,2,3)=\left(a_{2}-a_{1} M_{1} M_{2}\right)\left(t_{12}\right)^{-\Delta_{12,3} / 2}\left(t_{23}\right)^{-\Delta_{23,1} / 2}\left(t_{13}\right)^{-\Delta_{13,2} / 2} e^{i\left(\frac{M_{1}}{2} \frac{x_{13}^{2}}{t_{13}}+\frac{M_{2}}{2} \frac{x_{23}^{2}}{t_{23}}\right)} I
$$

where

$$
a_{2}=a_{1}\left[\Delta_{2} \Delta_{3}+\frac{1}{2}(d+2-\Delta)\left(\Delta_{23,1}\right)\right]
$$


and $I$ is the same integral as (4.6). The details of the evaluation of (4.6) are shown in an appendix. We note that this is the same integral which appeared in [12] where the nonrelativistic three-point functions in CFT where identified with response functions in Martin-Siggia-Rose theory, but the integral has not been explicitly evaluated there.

\section{B. Four-point functions}

Finally, we consider the four-point function arising from a bulk quartic interaction $\phi^{4}$

$$
A_{1}(1,2,3,4)=\int K_{1}^{*}\left(r, \vec{x}, t ; \vec{x}_{1}, t_{1}\right) K_{2}^{*}\left(r, \vec{x}, t ; \vec{x}_{2}, t_{2}\right) K_{3}^{*}\left(r, \vec{x}, t ; \vec{x}_{3}, t_{3}\right) K_{4}\left(r, \vec{x}, t ; \vec{x}_{4}, t_{4}\right) .
$$

Performing the same tricks as above we find

$$
A_{1}(1,2,3,4)=\int d \alpha_{1} d \alpha_{2} d \alpha_{3} e^{-i M_{1} \alpha_{1}} e^{-i M_{2} \alpha_{2}} e^{-i M_{3} \alpha_{3}} I_{1}
$$

where

$$
\begin{aligned}
I_{1}=b_{1} \int \frac{d r d^{d} x d t d \mu}{r^{d+3}}\left(\frac{r}{r^{2}+\left(x-x_{1}\right)^{2}+2\left(t-t_{1}\right)\left(\mu-\alpha_{1}\right)}\right)^{\Delta_{1}} \\
\quad \times\left(\frac{r}{r^{2}+\left(x-x_{2}\right)^{2}+2\left(t-t_{2}\right)\left(\mu-\alpha_{2}\right)}\right)^{\Delta_{2}} \\
\quad \times\left(\frac{r}{r^{2}+\left(x-x_{3}\right)^{2}+2\left(t-t_{3}\right)\left(\mu-\alpha_{3}\right)}\right)^{\Delta_{3}}\left(\frac{r}{r^{2}+\left(x-x_{4}\right)^{2}+2\left(t-t_{4}\right) \mu}\right)^{\Delta_{4}}
\end{aligned}
$$

is the same integral as in the relativistic AdS/CFT case [19, 20] and $b_{1}=\bar{c}_{\Delta_{1}} \bar{c}_{\Delta_{2}} \bar{c}_{\Delta_{3}} c_{\Delta_{4}}$.

Let us consider as an example the simple case, $\Delta_{1}=\Delta_{2}=\Delta_{3}=\Delta_{4}=\Delta$, for which it is known [20] that

$$
I_{1}=\frac{\Gamma\left(2 \Delta-\frac{d}{2}-1\right)}{\Gamma(2 \Delta)} \frac{2 \pi^{(d+2) / 2}}{\left(\chi_{12}^{2} \chi_{34}^{2}\right)^{\Delta}} \int_{0}^{\infty} d z_{2} F_{1}\left(\Delta, \Delta ; 2 \Delta ; 1-\frac{(\eta+\zeta)^{2}}{(\eta \zeta)^{2}}-\frac{4}{\eta \zeta} \sinh ^{2} z\right)
$$

where

$$
\eta=\frac{\chi_{12} \chi_{34}}{\chi_{14} \chi_{23}}, \quad \zeta=\frac{\chi_{12} \chi_{34}}{\chi_{13} \chi_{24}}
$$

are conformal cross-ratios and

$$
\chi_{i j}^{2}=\left(x_{i j}^{2}+2 t_{i j} \alpha_{i j}\right)
$$

Again introducing the regulator and making the change of variables $u_{i}=\alpha_{i}+\frac{x_{i 4}^{2}}{2 t_{i 4}}(1+i \epsilon)$, we have

$$
\chi_{i 4}^{2}=2 t_{i 4}(1-i \epsilon) u_{i}, \quad \chi_{i j}^{2}=2 t_{i j}(1-i \epsilon)\left(u_{i j}+v_{i j}(1+i \epsilon)\right) \quad \text { for } i, j<4
$$


where $v_{i j}$ is defined in (2.10) and $\eta$ and $\zeta$ change similarly.

Finally then we arrive at

$$
A_{1}(1,2,3,4)=\frac{\Gamma\left(2 \Delta-\frac{d}{2}-1\right)}{\Gamma(2 \Delta)} \frac{2 \pi^{(d+2) / 2}}{\left(4 t_{12} t_{34}\right)^{\Delta}} e^{i\left(\frac{M_{1}}{2} \frac{x_{14}^{2}}{t_{14}}+\frac{M_{2}}{2} \frac{x_{24}^{2}}{t_{24}}+\frac{M_{3}}{2} \frac{x_{34}^{2}}{t_{34}}\right)} I_{2}
$$

in terms of

$$
\begin{aligned}
I_{2}= & \int_{\mathcal{C}_{1}} d u_{1} \int_{\mathcal{C}_{2}} d u_{2} \int_{\mathcal{C}_{3}} d u_{3} e^{-i M_{1} u_{1}} e^{-i M_{2} u_{2}} e^{-i M_{3} u_{3}} \\
& \quad\left(u_{3}\left(u_{12}+v_{12}(1+i \epsilon)\right)\right)^{-\Delta} \int_{0}^{\infty} d z_{2} F_{1}\left(\Delta, \Delta ; 2 \Delta, 1-\frac{(\eta+\zeta)^{2}}{(\eta \zeta)^{2}}-\frac{4}{\eta \zeta} \sinh ^{2} z\right),
\end{aligned}
$$

where the contour $\mathcal{C}_{j}$ is $\left(\mathbf{R}+i \frac{x_{j 4}^{2}}{2 t_{j 4}} \epsilon\right)$. We see the result is consistent with the general four-point function (2.13).

We could treat the scalar exchange diagram similarly by first performing the Fourier transform and then doing the integral over the AdS bulk, leading to

$$
A_{2}(1,2,3,4)=\int d \alpha^{\prime} d \beta^{\prime} d \gamma^{\prime} d \mu^{\prime} e^{-i M_{1} \alpha^{\prime}} e^{-i M_{2} \beta^{\prime}} e^{i M_{3} \gamma^{\prime}} e^{i M_{4} \mu^{\prime}} I_{4},
$$

where the integral $I_{4}$ is well-known from the original AdS/CFT literature [21]

$$
\begin{array}{r}
I_{4}=\int \frac{d z d^{d} x d t d \eta}{z^{d+3}} \frac{d w d^{d} y d \tau d \eta^{\prime}}{w^{d+3}} K_{1}\left(z, \eta, \vec{x}, t ; \alpha^{\prime}, \vec{x}_{1}, t_{1}\right) K_{3}\left(z, \eta, \vec{x}, t ; \beta^{\prime}, \vec{x}_{3}, t_{3}\right) \\
G\left(z, \eta, \vec{x}, t ; w, \eta^{\prime}, \vec{y}, \tau\right) K_{2}\left(w, \eta^{\prime}, \vec{y}, \tau ; \gamma^{\prime}, \vec{x}_{2}, t_{2}\right) K_{4}\left(w, \eta^{\prime}, \vec{y}, \tau ; \mu^{\prime}, \vec{x}_{4}, t_{4}\right) .
\end{array}
$$

After a change of variables, the integral could be brought to the form (2.13) as required by non-relativistic conformal invariance.

\section{Acknowledgments}

We are grateful to K. Balasubramanian, A. Jevicki, K. Jin, C. Kalousios, S. Roy and especially M. Spradlin for useful conversations. This work was supported in part by the US Department of Energy under contract DE-FG02-91ER40688 and the US National Science Foundation under grant PHY-0643150 CAREER and PECASE.

\section{APPENDIX A: THREE-POINT FUNCTION INTEGRAL}

Here we consider the integral (4.6)

$$
\int_{\mathcal{C}_{1}} d u \int_{\mathcal{C}_{2}} d w e^{-i M_{1} u} e^{-i M_{2} w} \frac{1}{\left(u-w+v_{12}(1+i \epsilon)\right)^{\Delta_{12,3} / 2}} \frac{1}{w^{\Delta_{23,1} / 2} u^{\Delta_{13,2} / 2}}
$$


where the contour $\mathcal{C}_{j}$ is $\left(\mathbf{R}+i \frac{\vec{x}_{j 3}^{2}}{2 t_{j 3}} \epsilon\right)$ for $\epsilon>0$.

Let us consider the case $t_{23}>0$ and $t_{12}>0$. Combining the two denominators involving $u$ with a Feynman parameter $z$ leads to

$$
I=\frac{\Gamma\left(\frac{\Delta_{12,3}}{2}+\frac{\Delta_{13,2}}{2}\right)}{\Gamma\left(\frac{\Delta_{12,3}}{2}\right) \Gamma\left(\frac{\Delta_{13,2}}{2}\right)} \int_{0}^{1} d z z^{\frac{\Delta_{12,3}}{2}-1}(1-z)^{\frac{\Delta_{13,2}}{2}-1} \int_{\mathcal{C}_{2}} d w e^{-i M_{2} w} w^{-\Delta_{23,1} / 2} A
$$

where

$$
\begin{aligned}
A & =\int_{\mathcal{C}_{1}} d u \frac{e^{-i M_{1} u}}{\left[u+z\left(-w+v_{12}(1+i \epsilon)\right)\right]^{\frac{\Delta_{12,3}}{2}+\frac{\Delta_{13,2}}{2}}} \\
& =2 \pi \frac{\left(M_{1}\right)^{\frac{\Delta_{12,3}}{2}+\frac{\Delta_{13,2}}{2}-1}}{\Gamma\left(\frac{\Delta_{12,3}}{2}+\frac{\Delta_{13,2}}{2}\right)} \exp \left[i M_{1} z\left(-w+v_{12}(1+i \epsilon)\right)\right] \exp \left[-\frac{i \pi}{2}\left(\frac{\Delta_{12,3}}{2}+\frac{\Delta_{13,2}}{2}\right)\right],
\end{aligned}
$$

which we have evaluated by using the identity

$$
\int_{\mathbf{R}+i \alpha} d u \frac{e^{-i M u}}{(u+z)^{f}}=2 \pi \frac{M^{f-1}}{\Gamma(f)} e^{i z M} e^{-i \pi f / 2}, \quad f>0, M>0, \operatorname{Im}(z) \geq 0 .
$$

Noting that $\frac{\Delta_{12,3}}{2}+\frac{\Delta_{13,2}}{2}=\Delta_{1}$, the original integral then becomes

$$
I=\frac{2 \pi\left(M_{1}\right)^{\Delta_{1}-1} e^{-i \pi \Delta_{1} / 2}}{\Gamma\left(\frac{\Delta_{12,3}}{2}\right) \Gamma\left(\frac{\Delta_{13,2}}{2}\right)} \int_{0}^{1} d z z^{\frac{\Delta_{12,3}}{2}-1}(1-z)^{\frac{\Delta_{13,2}}{2}-1} e^{i M_{1} z v_{12}(1+i \epsilon)} B
$$

where

$$
B=\int_{\mathcal{C}_{2}} d w e^{-i M_{2} w} w^{-\Delta_{23,1} / 2} e^{-i M_{1} z w}=2 \pi \frac{\left(M_{2}+z M_{1}\right)^{\frac{\Delta_{23,1}}{2}-1}}{\Gamma\left(\frac{\Delta_{23,1}}{2}\right)} \exp \left[-\frac{i \pi}{2} \frac{\Delta_{23,1}}{2}\right] .
$$

Putting everything together then gives

$$
\begin{aligned}
& I=\frac{4 \pi^{2} M_{1}^{\Delta_{1}-1} M_{2}^{\frac{\Delta_{23,1}}{2}-1}}{\Gamma\left(\frac{\Delta_{12,3}}{2}\right) \Gamma\left(\frac{\Delta_{13,2}}{2}\right) \Gamma\left(\frac{\Delta_{23,1}}{2}\right)} \exp \left[-\frac{i \pi}{4} \Delta\right] \\
& \times \int_{0}^{1} d z z^{\frac{\Delta_{12,3}}{2}-1}(1-z)^{\frac{\Delta_{13,2}}{2}-1}\left(1+\frac{M_{1}}{M_{2}} z\right)^{\frac{\Delta_{23,1}}{2}-1} \exp \left[i M_{1} z v_{12}(1+i \epsilon)\right],
\end{aligned}
$$

and the final integral can be evaluated thanks to the identity [22]

$$
\int_{0}^{1} x^{\nu-1}(1-x)^{\lambda-1}(1+\beta x)^{-\rho} e^{-\mu x} d x=B(\nu, \lambda) \Phi_{1}(\nu, \rho, \lambda+\nu,-\beta,-\mu),
$$

which leads to the advertised result (4.6) in terms of the beta function

$$
B(x, y)=\int_{0}^{1} t^{x-1}(1-t)^{y-1} d t
$$


and the confluent hypergeometric function function

$$
\Phi_{1}(\alpha, \beta, \gamma, x, y)=\sum_{m, n=0}^{\infty} \frac{(\alpha)_{m+n}(\beta)_{m}}{(\gamma)_{m+n} m ! n !} x^{m} y^{n}
$$

[1] J. M. Maldacena, "The large N limit of superconformal field theories and supergravity," Adv. Theor. Math. Phys. 2, 231 (1998) [Int. J. Theor. Phys. 38, 1113 (1999)] arXiv:hep-th/9711200].

[2] S. S. Gubser, I. R. Klebanov and A. M. Polyakov, "Gauge theory correlators from non-critical string theory," Phys. Lett. B 428, 105 (1998) arXiv:hep-th/9802109.

[3] E. Witten, "Anti-de Sitter space and holography," Adv. Theor. Math. Phys. 2, 253 (1998) arXiv:hep-th/9802150].

[4] C. P. Herzog, A. Karch, P. Kovtun, C. Kozcaz and L. G. Yaffe, JHEP 0607, 013 (2006) arXiv:hep-th/0605158]. • S. S. Gubser, Phys. Rev. D 74, 126005 (2006) arXiv:hep-th/0605182]. • C. P. Herzog, JHEP 0609, 032 (2006) arXiv:hep-th/0605191. - J. J. Friess, S. S. Gubser and G. Michalogiorgakis, JHEP 0609, 072 (2006) arXiv:hep-th/0605292]. • H. Liu, K. Rajagopal and U. A. Wiedemann, JHEP 0703, 066 (2007) arXiv:hep-ph/0612168. • D. T. Son and A. O. Starinets, Ann. Rev. Nucl. Part. Sci. 57, 95 (2007) arXiv:0704.0240 [hep-th]]. • C. P. Herzog, P. Kovtun, S. Sachdev and D. T. Son, Phys. Rev. D 75, 085020 (2007) arXiv:hep-th/0701036. • S. A. Hartnoll, P. K. Kovtun, M. Muller and S. Sachdev, Phys. Rev. B 76, 144502 (2007) arXiv:0706.3215 [cond-mat.strel]]. • S. A. Hartnoll, C. P. Herzog and G. T. Horowitz, Phys. Rev. Lett. 101, 031601 (2008) arXiv:0803.3295 [hep-th]]. • S. S. Gubser, Gen. Rel. Grav. 39, 1533 (2007) [Int. J. Mod. Phys. D 17, 673 (2008)]. • S. S. Gubser and A. Karch, arXiv:0901.0935 [hep-th].

[5] K.M.O'Hara et al., arXiv:cond-mat/0212463 • C.A.Regal, M.Greiner, D.S.Jin arXiv:cond-mat/0401554 • M. Bartenstein et al., arXiv:cond-mat/0401109 • M.W. Zwierlein et al., arXiv:cond-mat/0403049 • J. Kinast et al., arXiv:cond-mat/0403540 • T. Bourdel et al., arXiv:cond-mat/0403091

[6] D. T. Son, "Toward an AdS/cold atoms correspondence: a geometric realization of the Schroedinger symmetry," Phys. Rev. D 78, 046003 (2008) [arXiv:0804.3972 [hep-th]]. 
[7] K. Balasubramanian and J. McGreevy, "Gravity duals for non-relativistic CFTs," Phys. Rev. Lett. 101, 061601 (2008) arXiv:0804.4053 [hep-th]].

[8] J. Maldacena, D. Martelli, Y. Tachikawa "Comments on string theory backgrounds with nonrelativistic conformal symmetry.," arXiv:0807.1100 [hep-th]

[9] A.Adams, K.Balasubramanian, J.McGreevy "Hot Spacetimes for Cold Atoms," arXiv:0807.1111 [hep-th]

[10] C.P. Herzog, M. Rangamani, S.F. Ross "Heating up Galilean holography.," arXiv:0807.1099 [hep-th]

[11] M. Alishahiha, R. Fareghbal, A. E. Mosaffa and S. Rouhani, arXiv:0902.3916 [hep-th]. • K. M. Lee, S. Lee and S. Lee, arXiv:0902.3857 [hep-th]. • A. Galajinsky and I. Masterov, arXiv:0902.2910 [hep-th]. • Y. Nakayama, arXiv:0902.2267 [hep-th]. • Y. Nakayama, M. Sakaguchi and K. Yoshida, arXiv:0902.2204 [hep-th]. • A. Bagchi and R. Gopakumar, arXiv:0902.1385 [hep-th]. - A. Akhavan, M. Alishahiha, A. Davody and A. Vahedi, arXiv:0902.0276 [hep-th]. • P. Horava, arXiv:0901.3775 [hep-th]. • M. Alishahiha and A. Ghodsi, arXiv:0901.3431 [hep-th]. • A. Donos and J. P. Gauntlett, arXiv:0901.0818 [hep-th]. • S. S. Pal, arXiv:0901.0599 [hep-th]. • U. H. Danielsson and L. Thorlacius, arXiv:0812.5088 [hep-th]. • M. Taylor, arXiv:0812.0530 [hep-th]. • A. Adams, A. Maloney, A. Sinha and S. E. Vazquez, arXiv:0812.0166 [hep-th]. • A. Akhavan, M. Alishahiha, A. Davody and A. Vahedi, arXiv:0811.3067 [hep-th]. • Y. Nakayama, S. Ryu, M. Sakaguchi and K. Yoshida, JHEP 0901, 006 (2009) [arXiv:0811.2461 [hep-th]]. • M. Rangamani, S. F. Ross, D. T. Son and E. G. Thompson, JHEP 0901, 075 (2009) arXiv:0811.2049 [hep-th]]. • L. Mazzucato, Y. Oz and S. Theisen, arXiv:0810.3673 [hep-th]. • M. Schvellinger, JHEP 0812, 004 (2008) arXiv:0810.3011 [hep-th]]. • S. A. Hartnoll and K. Yoshida, JHEP 0812, 071 (2008) arXiv:0810.0298 [hep-th]]. • F. L. Lin and S. Y. Wu, arXiv:0810.0227 [hep-th]. • D. Yamada, arXiv:0809.4928 [hep-th]. • C. Duval, M. Hassaine and P. A. Horvathy, arXiv:0809.3128 [hep-th]. • P. Kovtun and D. Nickel, Phys. Rev. Lett. 102, 011602 (2009) arXiv:0809.2020 [hep-th]]. • S. Pal, arXiv:0809.1756 [hep-th]. • S. Sekhar Pal, arXiv:0808.3232 [hep-th]. • S. S. Pal, arXiv:0808.3042 [hep-th]. • S. Kachru, X. Liu and M. Mulligan, Phys. Rev. D 78, 106005 (2008) arXiv:0808.1725 [hep-th]]. • A. V. Galajinsky, Phys. Rev. D 78, 087701 (2008) arXiv:0808.1553 [hep-th]]. • D. Minic and M. Pleimling, arXiv:0807.3665 [cond-mat.statmech]. • J. W. Chen and W. Y. Wen, arXiv:0808.0399 [hep-th]. • Y. Nakayama, JHEP 0810, 
083 (2008) arXiv:0807.3344 [hep-th]]. • W. Y. Wen, arXiv:0807.0633 [hep-th]. • M. Sakaguchi and K. Yoshida, JHEP 0808, 049 (2008) arXiv:0806.3612 [hep-th]]. • J. L. B. Barbon and C. A. Fuertes, JHEP 0809, 030 (2008) [arXiv:0806.3244 [hep-th]]. • W. D. Goldberger, arXiv:0806.2867 [hep-th]. • M. Sakaguchi and K. Yoshida, arXiv:0805.2661 [hep-th].

[12] M. Henkel, J. Unterberger, "Schrodinger invariance and space-time symmetries," arXiv:hep-th/0302187

[13] C. A. Fuertes and S. Moroz, "Correlation functions in the non-relativistic AdS/CFT correspondence," arXiv:0903.1844 [hep-th].

[14] Y. Nishida and D. T. Son, "Nonrelativistic conformal field theories," Phys. Rev. D 76, 086004 (2007) arXiv:0706.3746 [hep-th]].

[15] M. Henkel, "Schrodinger invariance in strongly anisotropic critical systems," J. Statist. Phys. 75, 1023 (1994) arXiv:hep-th/9310081.

[16] P. H. Ginsparg, "APPLIED CONFORMAL FIELD THEORY," arXiv:hep-th/9108028.

[17] E. D'Hoker and D. Z. Freedman, "General scalar exchange in AdS(d+1)," Nucl. Phys. B 550, 261 (1999) arXiv:hep-th/9811257.

[18] D. Z. Freedman, S. D. Mathur, A. Matusis and L. Rastelli, "Correlation functions in the CFT $(d) / \operatorname{AdS}(d+1)$ correspondence," Nucl. Phys. B 546, 96 (1999) arXiv:hep-th/9804058.

[19] E. D'Hoker and D. Z. Freedman, "Supersymmetric gauge theories and the AdS/CFT correspondence," arXiv:hep-th/0201253.

[20] W. Mueck and K. S. Viswanathan, "Conformal field theory correlators from classical scalar field theory on AdS(d+1)," Phys. Rev. D 58, 041901 (1998) arXiv:hep-th/9804035.

[21] H. Liu, A.A. Tseytlin, "On Four-point functions in the CFT/AdS Correspondce," arXiv:hep-th/9807097

[22] I. S. Gradshteyn, I. M. Ryshik: Table of Integrals, Series, and Products 